\title{
Design, Simulation and Fabrication of a Dielectrophoretic Separation Device Using Topographic Channel Structure
}

\author{
L. Zhang, F. Tatar, J.R. Mollinger, and A. Bossche \\ Lab. of Electronic Instrumentation, Dept. of Microelectronics \\ Delft University of Technology \\ Delft, the Netherlands \\ L.Zhang@tudelft.nl
}

\begin{abstract}
We present a novel device for continuous separation based on dielectrophoresis (DEP), where the non-uniform electric field is generated by applying a dc voltage over a circular channel structure instead of ac electrodes. The finite element simulation result shows it has the potential to separate particles of different sizes and dielectric properties in both spatial and time domain. Compared with the previously reported dieletrophoretic separation using electrode arrays [110], this structure is more mechanically robust and chemically inert. And compared with the previously reported electrodeless dielectrophoretic separation methods [11-14], this structure achieves high throughput and continuous separation.
\end{abstract}

\section{INTRODUCTION}

Modern particle separation techniques have been fundamental to many advances in cell biology, molecular genetics, biotechnological production, clinical diagnostics, and therapeutics [1]. A number of methods have been developed to separate particles, including the ubiquitous techniques of filtration, centrifugation, electrophoresis, and both fluorescence-(FACS) and magnetic-activated-cell sorting (MACS), taking advantage of differences in particle size, density, electrical charge, and specific immunological surface markers. As these technologies have reached maturity, it has become more difficult to make fundamental improvements in separation resolution, system complexity, sample size, and device cost. Therefore, novel physical methods by which different particle types may be effectively discriminated and selectively manipulated are desirable. To this end, particle dielectric properties have been explored through dielectrophoresis for developing particle separation techniques. As it is well known, the dielectrophoretic force arises due to a non-uniform electric field and the subsequent unbalanced action of the electrical force on a particle. Therefore, a precise manipulation of single particles can be realized by controlling the electric field without introducing mechanical movement.

In the past numerous dielectrophoretic methods including DEP migration [2,3], DEP affinity [4,5], dielectrophoretic field flow fractionation (DEP-FFF) $[1,6]$ and traveling wave dielectrophoresis (TWD) [7-10], the inhomogeneous ac electric fields are normally generated by various electrode geometries or electrode arrays. Those very small electrodes made to generate high gradient field, however, will cause gas evolution due to the electrolysis effect. In order to avoid this problem and make the structure more mechanically robust and chemically inert, works [11-14] are carried out to explore electrodeless DEP separation. However, none of these electrodeless DEP methods could achieve high throughput continuous separation. So, there comes up with a new demand for electrodeless DEP separation methods in a continuous manner. In this work, we present a circular channel structure which can realize continuous electrokinetic separation by electrodeless dielectrophoresis. The particles are transported electrokinetically by the electro-osmosis. They will continuously experience a DEP force due to the local non-uniform electric field inside the circular channel when moving towards the outlet. The particles of different sizes and dielectric properties will eventually get separated at the outlet. In order to prove the principle, the modeling and simulation were carried out to study the particle size, dielectric property and applied voltage level. The device has been successfully fabricated, and experiment results are expected to verify the design and the simulation method.

\section{THEORY}

The time averaged dielectrophoretic force acting on a spherical particle, immersed in a medium and exposed to a spatially non-uniform electric field can be described by [15]

$$
F_{D E P}=2 \pi \varepsilon_{m} R_{p}^{3} \operatorname{Re}[K(\omega)] \nabla E^{2}
$$

where $\nabla E^{2}$ is the gradient of electric field squared, $\varepsilon_{m}$ is the permittivity of the suspending medium, $R_{p}$ is the radius of the particle, and 


$$
K(\omega)=\frac{\varepsilon_{p}^{*}-\varepsilon_{m}^{*}}{\varepsilon_{p}^{*}+2 \varepsilon_{m}^{*}}
$$

$K(\omega)$ is the frequency dependent Claussius-Mosotti (CM) factor, $\varepsilon_{p}^{*}$ and $\varepsilon_{m}^{*}$ represent the frequency dependent complex permittivities of the particle and medium, respectively, The complex permittivity is defined as $\varepsilon_{p}^{*}=\varepsilon_{p}-j\left(\sigma_{p} / \omega\right)$ and $\varepsilon_{m}^{*}=\varepsilon_{m}-j\left(\sigma_{m} / \omega\right)$, where $j=\sqrt{-1}$, $\varepsilon$ is the permittivity, and $\sigma$ is the conductivity of the dielectric.

For small particles (low Reynolds numbers) ignoring Brownian motion and the buoyancy force, the DEP force moving the particle is balanced with the fluid drag force slowing it down, which is given by

$$
F_{D E P}=-F_{D r a g}=6 \pi \eta R_{p} v_{p}
$$

where $v_{p}$ is the particle velocity and $\eta$ is the medium viscosity. So that for a spherical particle the dielectrophoretic mobility will be

$$
\mu_{D E P}=\frac{R_{p}^{2} \varepsilon_{m} \operatorname{Re}[K(\omega)]}{3 \eta}
$$

in the direction of $\nabla E^{2}$. It can be seen that for a spherical particle moving in an electric field gradient, the dielectrophoretic mobility is proportional to the surface area of the particle and the $\mathrm{CM}$ factor and the direction of the particle movement is dependent on the sign of $\mathrm{CM}$ factor.

\section{DESIGN}

The principle of the continuous electrodeless DEP separation in circular channel is illustrated in Figure 1. By applying a dc voltage over the circular chamber, a nonuniform electric field will be generated whose gradient directs towards the centre of the circle. The sample mixture is driven along the channel by the electro-osmotic flow. Due to the different DEP force magnitudes and directions, the particles with different DEP responses move continuously to the different location across the channel as they flow, thus continuously separated into the different outlets. Furthermore, the particles following the outer side path will be left behind, since the electro-osmotic flow at the inner side of the channel is faster than that at the outer side of the channel.

In the micro-channels the Reynolds number is low enough for inertial effects to be irrelevant so that the centrifugal force on the particles could be ignored. Thus along the radius direction, the particle mobility depends on the CM factor and the particle size for a given suspending medium. The particles with positive DEP response $\operatorname{Re}[K(\omega)]>0$ will be driven away from the center. While the particles with negative DEP response $\operatorname{Re}[K(\omega)]<0$ will be attracted towards the center. As a result, the positive DEP particles will end up more inwards than the initial injection point while the negative DEP particles will end up more outwards than the initial injection point. For the particles with the same DEP polarity, the larger the particle is the higher mobility it has in the radius direction and the faster it will move towards the inner wall or outer wall of the channel. Therefore, this channel can be utilized to separate the particles of different DEP polarities and of the same DEP polarities but different sizes.

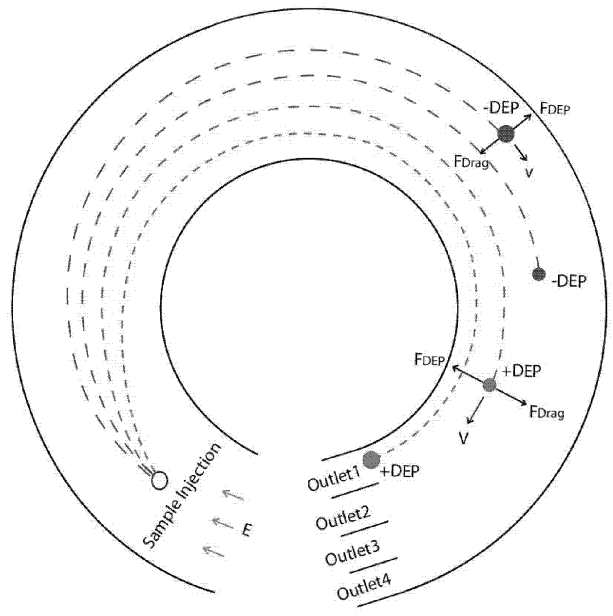

Figure 1. Schematic diagram of the dielectrophoretic separation in a circular micro-channel

Along the tangential direction, the low Reynolds number particles adapt to the fluid flow instantly. Since the velocity of the electro-osmotic flow on the sidewall is proportional to the electric field intensity, the flow velocity at the inner side of the channel where the highest electric field intensity exists will be faster than that at the outer side of the channel. Therefore, the time taken by particles with different sizes and dielectric properties to reach the outlets is different as shown in Figure 1.

\section{MOdELling AND SimUlation}

To simulate the particle behavior in the micro-circularchannel, the trajectory calculations are implemented in Matlab 7.0 after the field calculations are performed with Femlab 3.1i. The goal of the simulation is to prove the hypothesis introduced earlier in previous section, that the particles of different dielectric property can be separated in both spatial and time domain in the micro-channel.

In Femlab 3.1i, a 3D channel model enclosed by a $50 \mu \mathrm{m}$ inner circle and a $100 \mu \mathrm{m}$ outer circle is built and the height of the channel is $40 \mu \mathrm{m}$. A $10 \mathrm{~V} \mathrm{dc}$ voltage is applied over the channel. The simulation of electric field is shown in Figure 2 that the maximum field density is at the inner side of the channel. The dielectrophoretic force normalized to polarizablility $\nabla E^{2}$, depicted in Figure 3, orients towards the center point. The force magnitude drops as the radius increases. 


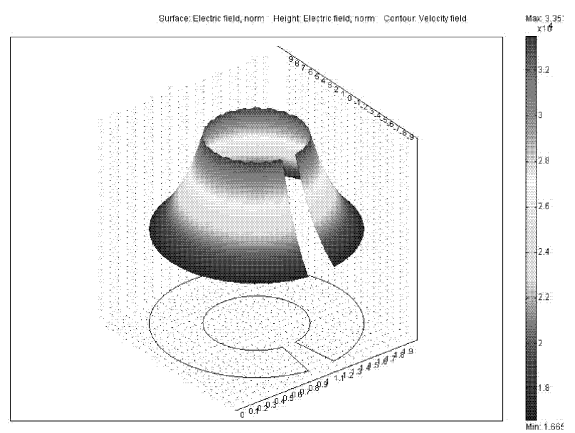

Figure 2. Simulation of Electric Field

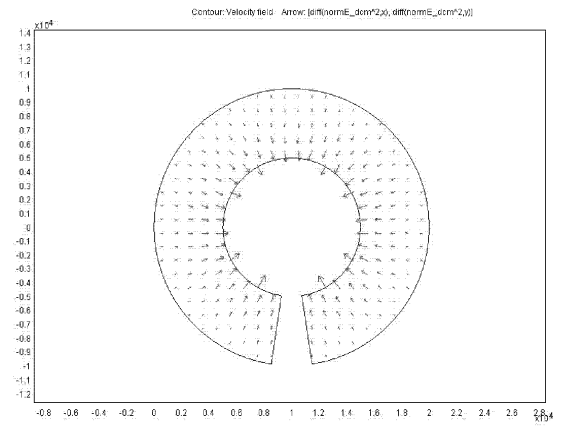

Figure 3. DEP force normalized to polarizability, $\nabla E^{2}$

For the sphere low Reynolds number particles moving in the uniform viscous flow with dielectrophoretic force acting on them, the velocity of the particles can be calculated as

$$
v_{\text {paricle }}=\frac{F_{D E P}}{6 \pi R_{p} \mu}+v_{\text {fluid }}
$$

The position of the particle after a certain time can be approximated by summing the distances traveled in a short period of time with the velocities calculated by above formula. Four particles of different size and dielectric properties are simulated, which are positive DEP particles with $5 \mu \mathrm{m}$ and $10 \mu \mathrm{m}$ radius, and negative DEP particles with $5 \mu \mathrm{m}$ and $10 \mu \mathrm{m}$, respectively. The simulation result for the angle displacement of these four particles is shown in Figure 4, which verifies the particle separation in the time domain.

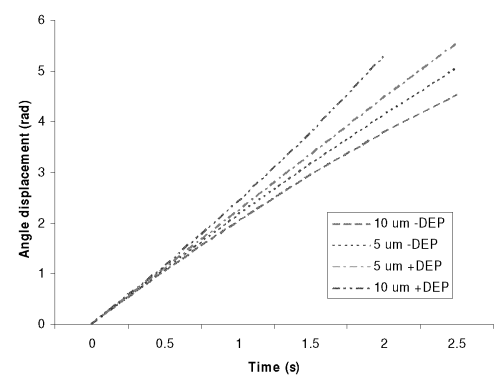

Figure 4. Simulation result for the angle displacement of 4 particles with different size and dielectric properties
Varying the voltage over the channel, we simulated the particle's ending position across the outlet. The result is shown in Figure 5, rin and rout represent the inner side point and the outer side point of the outlet respectively, from which we can see the separation in the spatial domain. With lower field intensity, the particles can be hardly separated due to the dramatically reduced DEP force which is proportional to the squared field gradient. While with higher field intensity, the greatly enlarged DEP force makes those particles over-separated with respect to the outlets.

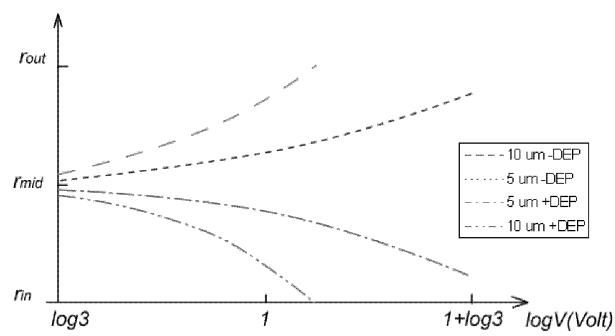

Figure 5. Particle positions across the outlets varying along the voltage applied over the circular channel

\section{FABRICATION}

The process flow is schematically depicted in Figure 6. A silicon wafer is used as a substrate. First, a thermal oxide layer is grown on both sides of the wafer (a). Additionally, a PECVD oxide layer is deposited on the back side of the wafer to be thick enough for silicon through-hole etching (b). Then, the titanium electrodes are formed for the actuator (c). The oxide layer on the back side of the wafer is patterned and etched to serve as a mask for through holes etching (d). The inlets and outlets are made by deep reactive ion etching through the silicon wafer (e). Sequentially, a thick layer of SU-8 is coated on to the top the silicon substrate and pattern to form the side wall of the channel (f). The oxide film on the top of the cavities is removed by wet etching $(\mathrm{g})$. Finally, the silicon substrate is aligned and bonded to a glass wafer using SU-8 adhesive bonding (h). The main part of the fabricated device is shown in Figure 7. The inlet for sample injection is located at the inner side of the separation channel instead of at the middle, which may have the application of separating negative DEP particles with different sizes.

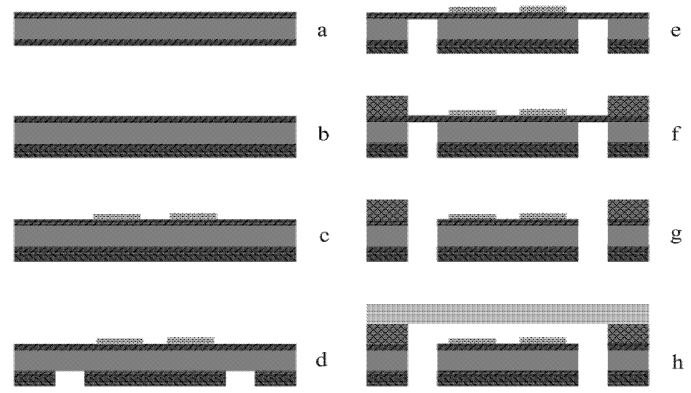

Figure 6. Process flowchart 
The $2 \mu \mathrm{m}$ thick thermal oxide layer is grown by wet oxidation at $1100^{\circ} \mathrm{C}$ for 8 hours and 11 minutes. The $4 \mu \mathrm{m}$ PECVD oxide layer is deposited from gas phase material $\mathrm{SiH} 4$ at $400^{\circ} \mathrm{C}$ by PECVD machine (Novellus C150mm). The $1 \mu \mathrm{m}$ thick titanium layer is deposited at $300^{\circ} \mathrm{C}$ with sputtering machine (TRIKON Sigma sputter coater) and the electrodes are formed by plasma etching machine (TRIKON Omega 201) with detected ending point. Through-holes are made by deep reactive ion etching at room temperature with the Bosch process (Adixen AMS100), which is shown in Figure 8.

Before SU-8 is applied onto the substrate, the wafer is baked at $100^{\circ} \mathrm{C}$ for 20 minutes on the hotplate to dehydrate the surface. To obtain a $40 \mu \mathrm{m}$ thick SU-8 layer, 30 second spin coating with the speed of $4000 \mathrm{rpm} / \mathrm{min}$ is performed. Soft bake at $95^{\circ} \mathrm{C}$ is performed for 20 minutes to evaporate the solvent and densify the film. To avoid the oxide film on the top of the cavity from break due to the vacuum chuck, which will cause wafer slipping, a layer of plastic foil might be sticked to the back side of the wafer before spin coating. The UV exposure is performed for 60 seconds by lithography machine (Electronic Vision EV420), and proximity mode with a $130 \mu \mathrm{m}$ separation gap is selected. Post exposure bake is performed for 5 minutes at $95^{\circ} \mathrm{C}$ to selectively cross-link the exposed portions of the SU-8 layer. Development is done by immersing the wafer into the SU-8 developer for around 8 minutes. Finally, the wafer is put onto the spinner and spun at a very high speed with some fresh developer on the top to complete rinsing and drying.

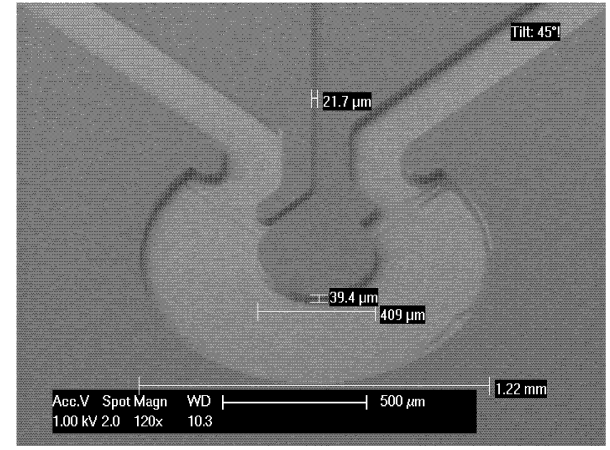

Figure 7. SEM picture of the separation channel with actuating electrodes

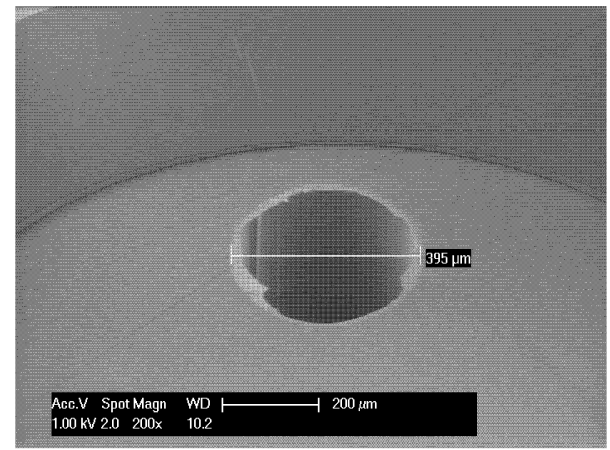

Figure 8. An inlet/outlet through-hole of $400 \mu \mathrm{m}$ diameter etched with DRIE Bosch process
SU-8 adhesive bonding is accomplished in five steps. 1. A $10 \mu \mathrm{m}$ thick SU-8 is spun on a dummy wafer, which will serve as the glue for bonding. The solvent has to be evaporated from the SU-8 layer on the hotplate. 2. The silicon substrate is pressed against the dummy wafer with the front side facing to the dummy wafer. And take it off after a while. 3. Align and pre-bond wafers on the hotplate. Pressure has to be applied to obtain uniform bonding. 4. Flood exposure through the top glass wafer to cross-link the SU-8 glue. 5. Post exposure bake is necessary for stress removal.

\section{CONCLUSION}

We have shown in this work design, simulation and fabrication of a novel dielectrophoretic separation device using circular channel structure. The simulation results have shown the separation in both time and spatial domain for the particles with different sizes and dielectric properties. The device has been successfully fabricated as shown in Figure 7. The experiment is expected to be carried out soon to verify the design and the simulation method.

\section{ACKNOWLEDGMENT}

This research is under grants from The Dutch Technology Foundation (STW), Applied Science Foundation of NWO and the technology programme of Ministry of Economic Affairs, the Netherlands.

\section{REFERENCES}

[1] X. Wang, J. Yang, Y. Huang, J. Vykoukal and P.R.C. Gascoyne, Anal. Chem., vol. 72, pp. 832-839, 2000

[2] P.R.C. Gascoyne, Y. Huang, R. Pethig, J. Vykoukal, F.F. Becker, Meas. Sci. Tchnol., vol. 3, pp. 439-445, 1992.

[3] N.G. Green, H. Morgan, J. Phys. D: Appl. Phys., vol 31, pp. L25L30, 1998.

[4] P.R.C. Gascoyne, X. Wang, Y. Huang, F.F. Becker, IEEE Trans. Ind Appl., vol. 33, pp. 670-678, 1997.

[5] F.F. Becker, X. Wang, Y. Huang, R. Pethig, J. Vykoukal, P.R.C. Gascoyne, Proc. Natl. Acad. Sci. U.S.A., vol. 92, pp. 860-864, 1995.

[6] X. Wang, J. Vykoukal, FF. Becker, P.R.C. Gascoyne, Biophy. J., vol. 74, pp. 2689-2701, 1998

[7] M.P. Hughes, X. Wang, J.P.H. Burt, R. Pethig, L.R. Watkins, Proceedings of 2nd International Conference on Comp. Electromag. vol. 384, pp. 48-51, 1994.

[8] M.S. Talary, J.P.H. Burt, J.A. Tame, R. Pethig, J. Phys. D: Appl Phys., vol. 29, pp. 2198-2203, 1996.

[9] H. Morgan, N.G. Green, M.P. Hughes, W. Monaghan, T.C. Tan, J. Micromech. Microeng., vol. 7, pp. 65-70, 1997.

[10] N.G. Green, M.P. Hughes, W. Monaghan, H. Morgan, Microelectr. Eng., vol. 35, pp. 421-424, 1997.

[11] C.F. Chou, J.O. Tegenfeldt, O. Bakajin, S.S. Chan, E.C. Cox, Biophy. J., vol. 83, pp. 2170-2179, 2002.

[12] C.F. Chou and F. Zenhausern, IEEE Eng. in medicine and biology magazine, pp. 62-67, 2003

[13] E.B. Cummings and A.K. Singh, Anal. Chem., vol. 75, pp. 4724 4731, 2003.

[14] B.H. Lapizco-Encinas, B.A. Simmons, E.B. Cummings, and Y. Fintschenko, Anal. Chem., vol. 76, pp. 1571-1579, 2004.

[15] T.B. Jones, 1995, Electromechanics of Particles, Cambidge University Press, Cambridge. 